\title{
Bioremediation potential of a halophilic bacterium Chromohalobacter salexigens EG1QL3: exopolysaccharide production, crude oil degradation, and heavy metal tolerance
}

Fedonenko Y.P. ${ }^{1}$, Ibrahim I.M. ${ }^{2}$, Sigida E.N. ${ }^{1}$, Safronova V.I. ${ }^{3}$, Kokoulin M.S. ${ }^{4}$, Muratova A.Y. ${ }^{1}$, Konnova S.A. ${ }^{1,5}$

${ }^{1}$ Institute of Biochemistry and Physiology of Plants and Microorganisms, Russian Academy of Sciences, Saratov, Russia; ${ }^{2}$ Fayoum University, Fayoum, Egypt; ${ }^{3}$ All-Russian Research Institute for Agricultural Microbiology, Saint Petersburg, Russia; ${ }^{4}$ G.B. Elyakov Pacific Institute of Bioorganic Chemistry, Far Eastern Branch of Russian Academy of Sciences, Vladivostok, Russia; ${ }^{5}$ Chernyshevsky Saratov State University, Saratov, Russia E-mail: fedonenko_yu@ibppm.ru

Key message. Based on biochemical and phylogenetic analyses, isolated from a salt sample from Lake Qarun (Egypt) a halophilic strain EG1QL3 was identified as Chromohalobacter salexigens. The abilities of EG1QL3 to produce an extracellular polysaccharide, degrade oil, and resist to heavy metals were revealed.

Keywords: Chromohalobacter salexigens, extracellular polysaccharide, crude oil degradation, heavy metal tolerance

Hypersaline environments of natural and artificial origin, such as saline lakes, salt marshes, and solar evaporation ponds, are used widely for the production of salt, potash, and soda. A side effect of the industrial use of such environments is their pollution by oil compounds. To survive under unfavorable conditions, halophilic bacteria produce large quantities of protective extracellular biopolymers, with the predominance of proteins and exopolysaccharides (EPS).

A halophilic bacterial strain, EG1QL3, was isolated from a salt sample from Lake Qarun, Fayoum Province, Egypt. Morphological, physiological, biochemical, and phylogenetic analyses indicated that the strain belongs to the species Chromohalobacter salexigens. Strain EG1QL3 produced an EPS, with production peaking (14 g L $\left.{ }^{-1}\right)$ during growth on S-G medium containing $3 \%(\mathrm{w} / \mathrm{v})$ sucrose and $15 \%(\mathrm{w} / \mathrm{v}) \mathrm{NaCl}$ at $25{ }^{\circ} \mathrm{C}(\mathrm{pH} 8.0)$. For identifying the optimal conditions for EPS production, $C$. salexigens EG1QL3 was grown at various temperatures, $\mathrm{pH}$ values, and salt concentration. The composition of and the ratio between carbon sources was also varied. The EPS was precipitated from the culture broth with cold ethanol and was purified by gel filtration and anion-exchange chromatography. The molecular mass of the EPS was $1.1-0.7 \times 10^{5}$ Da. Diffuse reflectance infrared Fourier transform and nuclear magnetic resonance spectra showed that the EPS was a linear $\beta$ $(2 \rightarrow 6)$-linked fructan (levan). The EPS demonstrated emulsifying activity $\left(\mathrm{E}_{24}, \%\right)$ against kerosene $(15.6 \pm 0.5 \%)$, and sunflower oil $(38.5 \pm 0.7 \%)$. Furthermore, the prepared emulsions were stable for 7 days at $25^{\circ} \mathrm{C}$.

Strain $C$. salexigens EG1QL3 was able to utilize crude oil as the sole carbon source within 12 days causing it to coagulate. The best oil degradation values by $C$. salexigens EG1QL3 $(30 \%)$ were in the presence of $2.5 \mathrm{M} \mathrm{NaCl}$. The minimum inhibitory concentrations of heavy metals for strain EG1QL3 were $1.5 \mathrm{for} \mathrm{Zn}$ (II) and $2.0 \mathrm{mM}$ for each of $\mathrm{Cd}(\mathrm{II}), \mathrm{Pb}(\mathrm{II}), \mathrm{Ni}(\mathrm{II})$, and $\mathrm{Cu}(\mathrm{II})$. C. salexigens EG1QL3 expressed plant growth attributes. The strain grew on nitrogen-free medium, which indicated the presence of nitrogen-fixing ability, and produced siderophores during growth on CAS agar.

In present work, we characterized the structure of the strain's EPS and optimized conditions for its production. Strain $C$. salexigens EG1QL3 was found to be promising for use in the cleanup of saline environments contaminated with oil hydrocarbons and heavy metals. 